\title{
Comparative Analysis of Masticatory Performance and Masticatory Efficiency of Bilateral and Unilateral Mastication
}

\author{
Yun Mi-Yeon ${ }^{1}$, Jong-Hwa Jang ${ }^{1,2}$ \\ ${ }^{1}$ Department of Public Health, Graduate School, Dankook University, Korea \\ ${ }^{2}$ Department of Dental Hygiene, College of Public Health Sciences, Dankook University, Korea
}

\begin{abstract}
Objective: This study aimed to compare the differences in masticatory performance (MP) and masticatory efficiency (ME) between bilateral masticators and habitual unilateral masticators, and then inquired into whether there is a significant difference in MP and ME when leading habitual unilateral masticators into bilateral chewing for a certain period of time.

Methods: Participants included 25 bilateral masticators and 25 habitual unilateral masticators after selecting men and women of being distributed to 20-39 years old among those who do not have lost teeth \& orthodontic appliances and have third molar without occlusion. A method was compared by calculating MP and ME using a method (pulverization sample measurement) that judges the chewing efficiency with a level of pulverizing specimen. The unilateral masticators in the experimental group were conducted a test with the primary pulverization sample measurement and then were allowed to practice the chewing exercise with the non-chewing side for 30 minutes by 3 times a day, respectively, through being provided $1 \mathrm{~g}$ of Xylitol gum each in 21 pieces during 2 weeks before being carried out the secondary pulverization sample measurement method. Statistical analysis was analyzed by paired t-test and independent $t$-test using SPSS.

Results: A comparative analysis of the main chewing side before attracting the bilateral chewing in habitual unilateral masticators versus the main chewing side after driving the bilateral chewing: The average value of the and main chewing side after driving the bilateral chewing was high $(p<0.05)$. A comparative analysis of the non-chewing side before driving the bilateral chewing in habitual unilateral masticators versus the non-chewing side after prompting the bilateral chewing: The average value of the and non-chewing side after prompting the bilateral chewing was high $(\mathrm{p}<0.05)$.

Conclusions: In light of this outcome, the bilateral masticators were more efficient in MP and ME than the habitual unilateral masticators. The main chewing side in habitual unilateral masticators was more efficient in MP and ME than the non-chewing side.
\end{abstract}

Keywords: Masticatory performance, Masticatory efficiency, Habitual unilateral chewing, Bilateral masticator

Copyright (C) 2021. Korean Academy of Preventive Dentistry. All rights reserved.

This is an Open Access article distributed under the terms of the Creative Commons Attribution Non-Commercial License (http://creativecommons.org/licenses/ by-nc/4.0) which permits unrestricted non-commercial use, distribution, and reproduction in any medium, provided the original work is properly cited. 